Holmes GL, Children's Hospital, Boston, in an editorial (Ann Neurol 1994;35:509), appears to favor a trial period of withdrawal rather than indefinite continuation of AEDs, even in children with significant risk factors. He cites Lennox and Lennox (1960) who criticized proponents of anticonvulsant continuance for life, and notes the psychological consequences and expense of long term therapy. In contrast to the concerns of Shinnar and colleagues, Holmes down plays AED side effects as a compelling reason to discontinue therapy and finds that cognitive and behavioral impairments are overstated. He takes issue with the provocative statement of our respected colleague, John Freeman, that "antiepileptic drugs are all poisons." (Curr Probl Pediatr April 1994;24:139-48).

\title{
VALPROATE-INDUCED THROMBOCYTOPENIA
}

A 14-year-old mentally retarded boy with seizures who presented with severe thrombocytopenia, macrocytic anemia and allergic dermatitis after treatment with valproate for 12 years is reported from the University of Louvain Medical School, Brussels, Belgium. Serum valproate level was 48 $\mathrm{mcg} / \mathrm{ml}$. Bone marrow examination showed myeloblastic abnormalities. Recovery followed withdrawal of valproate. (Brichard B et al. Haematological disturbances during long-term valproate therapy. Eur I Pediatr May 1994;153:378-380). (Respond: Dr B Brichard, Dept Paediatric Haematology, Univ Louvain Med School, Avenue Hippocrate 10, B-1200 Brussels, Belgium).

COMMENT. Thrombocytopenia is a well known side effect of valproate therapy. It is related to antibody-mediated platelet destruction, and tests for serum direct antiplatelet antibodies are positive. This report is unusual in the delayed occurrence of hematologic toxicity, even with relatively low serum levels of valproate.

\section{SEIZURE DISORDERS}

\section{FIRST FEBRILE SEIZURE CHARACTERISTICS}

Clinical characteristics of 910 first febrile seizures in children aged 8 to 34 months, evaluated by telephone interview of parents, are reported from the University of Washington School of Medicine, Seattle, WA. A male preponderance of $57 \%$ and a family history of febrile seizures in $29 \%$ were elicited. Focal seizures, including only eye deviation in the definition of some, were reported in 18\%; Todd's paresis in $4 \%$. Infections associated with fever included otitis media $32 \%$, tonsillitis or URI $12 \%$, viral exanthem $12 \%$, and immunizations $2 \%$. The average temperature recorded at the time of the seizure was $103.7^{\circ} \mathrm{F}$. Prolonged seizures and recurrence in the same illness, factors related to increased risk of subsequent nonfebrile seizures, were significantly more frequent in children aged 8-11 months, when compared to those older than 12 months. (Farwell JR et al. First febrile seizure. Characteristics of the child, the seizure, and the illness. Clin Pediatr May 1994;33:263-267). (Respond: Jacqueline R Farwell MD, Division of Neurology, Children's Hospital, 4800 Sand Point Way NE, Seattle, WA 98105).

COMMENT. Notwithstanding the limitations of the method of data collection, some of the Seattle findings are of interest as they compare 
with numerous previous reports of similar clinical febrile seizure studies. Between 1924 and 1965, 51 articles involving approximately 10,000 febrile seizure patients were published in the world literature. (Millichap JG. Febrile Convulsions, New York, Macmillan, 1968). A male preponderance was established in 29 series, with a mean sex ratio of 1.4 to 1 , a family history of febrile seizures was found in $30 \%$, and the mean threshold convulsive temperature was $104.0^{\circ} \mathrm{F}$. In contrast to the Seattle findings, otitis media accounted for only $2.9 \%$ of associated fevers, and tonsillitis or pharyngitis was by far the most frequent illness, occurring in $59 \%$ of febrile episodes. Focal seizures were reported in a mean of $11 \%$, and $14 \%$ in one prospective study. The incidence of Todd's paresis was $3.7 \%$ and similar to that observed in Seattle. Of prognostic importance is the confirmation of age at onset $(<1$ year) as a risk factor for complex febrile seizures in this study.

\section{EPILEPSY WITH MYOCLONIC ABSENCES}

Resistance to therapy and learning disabilities are stressed as frequent complications of the syndrome of "epilepsy with myoclonic absences" reported in 8 children, ages 6 to 16 years, seen at the University Hospital of Wales in a 10 year period. The mean age at onset was 4.9 years. Febrile seizures had occurred in siblings of 3 patients. Myoclonic absences were brief, and they could be precipitated by hyperventilation. Loss of awareness was associated with bilateral jerking of the head and upper limbs, and the EEG showed rhythmic $3 \mathrm{c} / \mathrm{s}$ spike-wave discharges. The majority had generalized tonicclonic or astatic seizures and cerebellar ataxia in addition. All became learning disabled and 7 had behavioral problems, including restlessness and impulsiveness. Treatment with lamotrigine and valproate was partially effective. (Manonmani V, Wallace SJ. Epilepsy with myoclonic absences. Arch Dis Child April 1994;70:288-290). (Respond: Dr Wallace, Dept Paediatric Neurology, University Hospital of Wales, Heath Park, Cardiff CF4 4XW, Wales, UK).

COMMENT. The response to lamotrigine (LTG) reported by Wallace in this and a previous report is in contrast to the experience of Schlumberger E et al at the Hopital Saint Vincent de Paul, Paris, France. (Epilepsia

March/April 1994;35:359-367). Of 9 patients with myoclonic absence epilepsy treated with LTG, none was seizure-free at 3 months, 4 improved and 5 were unchanged. Side effects included skin rash, especially when LTG was added to VPA therapy, ataxia, drowsiness, and vomiting. The differentiation of this syndrome from typical absence epilepsy is important because of the poor response to treatment and the unfavorable long term outcome.

\section{GENETICS OF EARLY CHILDHOOD ABSENCE EPILEPSY}

The clinical and EEG family data of 140 cases of early childhood epilepsy with absences selected from the epilepsy family archive are reported from the Neuropaediatric Department of the University of Kiel, Germany. Patients with absences manifesting between the 1st and 5th year of age were selected for study. Two groups were formed: 1) GTCS, those presenting with generalized tonic-clonic seizures (90 cases); and 2) non-GTCS, presenting with absences (50 cases). GTCS at onset were afebrile or febrile. In $43 \%$ of probands absence were combined with myoclonic and/or myoclonic astatic seizures. Parents and their sibs of group 1 had seizures twice as often as parents and sibs in group 2 . The 